\title{
Low Momentum Muon Identification in the ATLAS Detector at the LHC
}

\author{
S. Tarem and N. Panikashvili
}

\begin{abstract}
Muon identification and high momentum measurement accuracy are crucial to fully exploit the physics potential of the ATLAS experiment at the LHC. The muon detection system of the ATLAS detector is characterized by two high precision tracking systems, namely the Inner Detector and the Muon Spectrometer, and sufficient calorimetry to ensure the safe absorption of hadrons before the spectrometer, yielding high purity muons with momenta above $3 \mathrm{GeV}$. In order to reconstruct and efficiently identify muon tracks, the MOORE and MuId Object-Oriented software packages have been developed within the ATLAS ATHENA framework. The MOORE and MuId algorithms combine to identify track segments in the Muon Spectrometer, exploit calorimeter information in order to extrapolate muons back to their production vertex, associate them with corresponding segments in the Inner Detector, and perform a combined fit to obtain optimal parameter resolution. To identify low $\mathrm{p}_{\mathrm{T}}$ muons that do not have a reconstructed Muon Spectrometer track, other strategies must be employed. We report on a method we developed to identify low $p_{T}$ muons and describe the offline performance studies. The simplicity of the algorithm renders it suitable for use in the ATLAS High-Level Trigger (HLT) system. We will discuss the adaptation of this algorithm for the Level Two Trigger.
\end{abstract}

Index Terms-ATLAS, LHC, muon, reconstruction.

\section{INTRODUCTION}

$\mathbf{M}$ UON identification and high accuracy in momentum measurement are crucial to fully exploit the physics potential of the ATLAS experiment at the LHC. Events with high momentum muons in the final state are among the most promising and robust signatures of "new physics" at LHC. Lower momentum muons, especially those from $\mathrm{J} / \psi$ decays, are an important signature for b-physics.

The muon detection system of the ATLAS detector is characterized by two high precision tracking systems, the Inner Detector (ID) [1] and the Muon Spectrometer (MS) [1]. There is sufficient calorimeter depth between them to ensure the absorption of hadrons before the spectrometer, yielding high purity muons with momenta above $3 \mathrm{GeV}$.

The ATLAS Muon Spectrometer has been designed to achieve momentum measurement with high efficiency and good resolution over a wide range of transverse momentum, pseudorapidity and azimuthal angle. Momentum measurement

Manuscript received November 11, 2004; revised March 31, 2006. This work was supported in part by the Israel Science Foundation, administered by the Israel Academy of Science and Humanities and the German Israeli Bi-national Science Foundation (GIF).

S. Tarem is with the Department of Physics, Technion-Israel Institute of Technology, Haifa, Israel (e-mail: tarem@ @echunix.technion.ac.il).

N. Panikashvili is with the CERN the from Department of Physics, Technion-Israel Institute of Technology, Haifa, Israel (e-mail: natalia. panikashvili@cern.ch).

Digital Object Identifier 10.1109/TNS.2006.875199

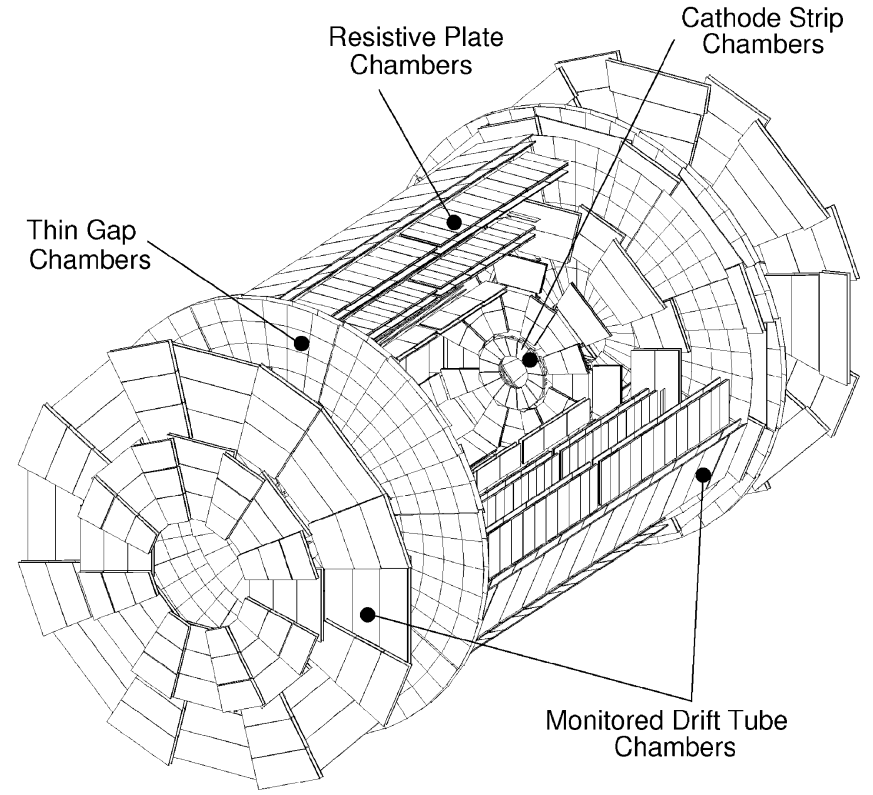

Fig. 1. Layout of the ATLAS Muon Spectrometer.

is performed via the magnetic deflection of muon tracks in a system of three large superconducting air-core toroid magnets instrumented with chambers dedicated to trigger, Resistive Plate Chambers (RPC) and Thin Gap Chambers (TCG), and with high precision tracking chambers, the Monitored Drift Tubes (MDT) and Cathode Strip Chambers (CSC). The layout of the ATLAS MS presented in Fig. 1. The Muon spectrometer consists of three measurement stations each providing a track segment in the precision measurement. The MDT chambers only provide a measurement in the $\eta$ direction. The trigger chambers are in the middle and outer station in the barrel, and in the inner and middle stations in the end-cap, and provide faster, less accurate $\eta$ and $\varphi$ measurements. The $\varphi$ measurements from the trigger chambers are used in the transformation of the MDT chamber signals to accurate digits.

In order to reconstruct and efficiently identify muon tracks, the Muon Object-Oriented Reconstruction (MOORE) and Muon Identification (MuId) software packages have been developed within the ATLAS ATHENA framework [3]. Those software packages are describes in [4] and [5]. MOORE identifies track segments using local pattern recognition at the chamber level in each of the three stations of the MS and performs a track fit, based on the package developed for the ID, iPatRec [6]. The result of MOORE is a collection of data objects that describe the reconstructed tracks at the entrance of the MS. 
The MuId algorithm associates muon tracks, which were reconstructed in the MS, with the corresponding reconstructed tracks in the ID. A common fit of the tracks in the ID and the MS provides a combined track with the optimal resolution obtainable with both systems.

Muons with low $\mathrm{p}_{\mathrm{T}}$ lose a significant part of their energy in the Calorimeter, and thus some of them cannot be reconstructed in the MS either because there are not sufficient digits for the track fit, or because the reconstructed segments, after having lost energy and affected by multiple scattering, don't match well to the Inner Detector tracks. The identification of these low $\mathrm{p}_{\mathrm{T}}$ muons is important for the b-physics capabilities of ATLAS. Up to $70 \%$ of $\mathrm{J} / \psi$ decays may be lost after passing the single muon trigger, because one of the muons has low $\mathrm{p}_{\mathrm{T}}$, and is not reconstructed in the MS. To identify these muons that do not have a reconstructed MS track, other strategies must be employed. We report on a method we developed to identify low $\mathrm{p}_{\mathrm{T}}$ muons and describe the offline performance studies. The simplicity of the algorithm renders it suitable for use in the ATLAS High Level Trigger system, in addition to the use in offline reconstruction. We discuss the adaptation of this algorithm for the ATLAS Second-Level Trigger (level-2). More details on the design of the ATLAS Trigger system can be found here [7].

\section{AlgORITHM}

The identification of low $\mathrm{p}_{\mathrm{T}}$ muons may use muon digits in a variety of sub detectors. This note describes a method that uses digits, a data associated with one detector channel, in all the sub detectors of the MS. Another method for identifying low $\mathrm{p}_{\mathrm{T}}$ muons relies on the part of the ATLAS Hadron Calorimeter that is made of scintillating tiles [8].

Inner detector tracks are extrapolated to the MS sub detectors, and there the digits of the muon sub-detectors are associated with them according to their proximity in $\eta$ and $\varphi$. We use digits from the MDT inner and middle stations, the CSC, the RPC middle station, and TGC. We also include the $\eta$ digits of the trigger chambers, which are not used in MOORE. The advantage of using all possible pieces of information is that muons may be identified even when a specific part of a sub-detector is malfunctioning.

This method was found to be efficient and provides good purity for identifying a second low $\mathrm{p}_{\mathrm{T}}$ muon resulting from $\mathrm{J} / \psi$ decays, when one muon from the decay was reconstructed in the MS.

The user may a-priori select to search for $\mathrm{J} / \psi$, or for events containing two muons, or for any muon. The output of the algorithm is a list of muon candidates, containing for each track the number of digits associated, and the distance between projected track and the closest digit in $\eta$ and in $\varphi$ in each MS sub-detector. The user will make the selection appropriate for her/his analysis, based on the information provided. Selection examples will be provided where we present the algorithm performance.

\section{PROGRAM}

The low $\mathrm{p}_{\mathrm{T}}$ muon identification starts after the MOORE and MuId reconstruction were performed. It follows these steps.

A new collection for MuId muon objects is created in which resulting muon candidates will be stored. MuId muon objects constructed by the MuId program described above are fetched. Each MuId object is inserted into the new collection. Information on associated digits in the MS sub-detector is appended to each candidate. Later ID tracks which were not identified by MuId are added to the collection if they are identified by this algorithm as muon candidates.

The ID track corresponding to the MuId object is identified and extrapolated to the MS. Propagation through the solenoidal field is performed to obtain $\phi$ at the spectrometer entrance. A parameterization of the toroidal field effects is used to extrapolate $\eta$ to the middle MS station. The extrapolation is done using the magnetic field description. Energy loss and multiple scattering effects are taken into account in the width of the road opened around the ID track.

Fig. 2 shows the distribution of distances in $\eta$ between the extrapolated ID track and TGC digits from the middle station, for $\mu^{+}$(dashed line) and $\mu^{-}$(dotted line). The top graph shows the raw difference without the toroidal field extrapolation, the middle graph shows the fit of the parameterization function, and the bottom plot shows this distance after the extrapolation.

We then attempt to associate each ID track under consideration with digits in each of the MS sub detectors. An association cut was defined for each sub detector (Table I); we count the number of digits passing the cut and record the closest digit to the extrapolated track trajectory. In the initial testing period we collect all the associated digits.

After we have gone over the whole list of MuId objects we proceed to do the same for all other ID tracks. These tracks, which have not been identified as muons by MOORE and MuId, are only included as muon candidates if MS digits were associated with them. The new list contains candidates that have either been identified by MuId, or had at least one MS digit associated with them.

Once we have finished processing tracks we check the results against user requirements. We calculate the invariant mass of each pairs of oppositely charged tracks that are in the collection. If it is within a given window, both muon candidates are marked as $\mathrm{J} / \psi$ decay candidates. If the user asks for $\mathrm{J} / \psi$ only, we check that there are two muons that are $\mathrm{J} / \psi$ candidates. If the user asked for di-muons we check that there are two muon candidates. If the user asks for any muons we check that the container is not empty. If the requirement is not met, the container is deleted.

\section{DATASETS USED}

Two samples of simulated events were used in order to develop the program and test its performance. A signal dataset $\Lambda_{\mathrm{b}} \rightarrow \Lambda \mathrm{J} / \psi$ with $\mathrm{J} / \psi \rightarrow \mu^{+} \mu^{-}$where one muon has $\mathrm{p}_{\mathrm{T}}>4 \mathrm{GeV}$ and the second muon has $\mathrm{p}_{\mathrm{T}}>2.5 \mathrm{GeV}$ was used to represent all $\mathrm{J} / \psi \rightarrow \mu^{+} \mu^{-}$channels, since the inclusive dataset was not simulated yet. The simulation was done without taking into account pile-up effects and cavern background. Thus the results of this study are more directly applicable for B-physics studies which will be performed at low luminosities below about $2 \times 10^{33} \mathrm{~cm}^{-2} \mathrm{~s}^{-1}$. At such low luminosities, below about $2 \times 10^{33} \mathrm{~cm}^{-2} \mathrm{~s}^{-1}$, events will be triggered by a single level-1 muon with $\mathrm{p}_{\mathrm{T}}$ threshold of about $6 \mathrm{GeV}$. 

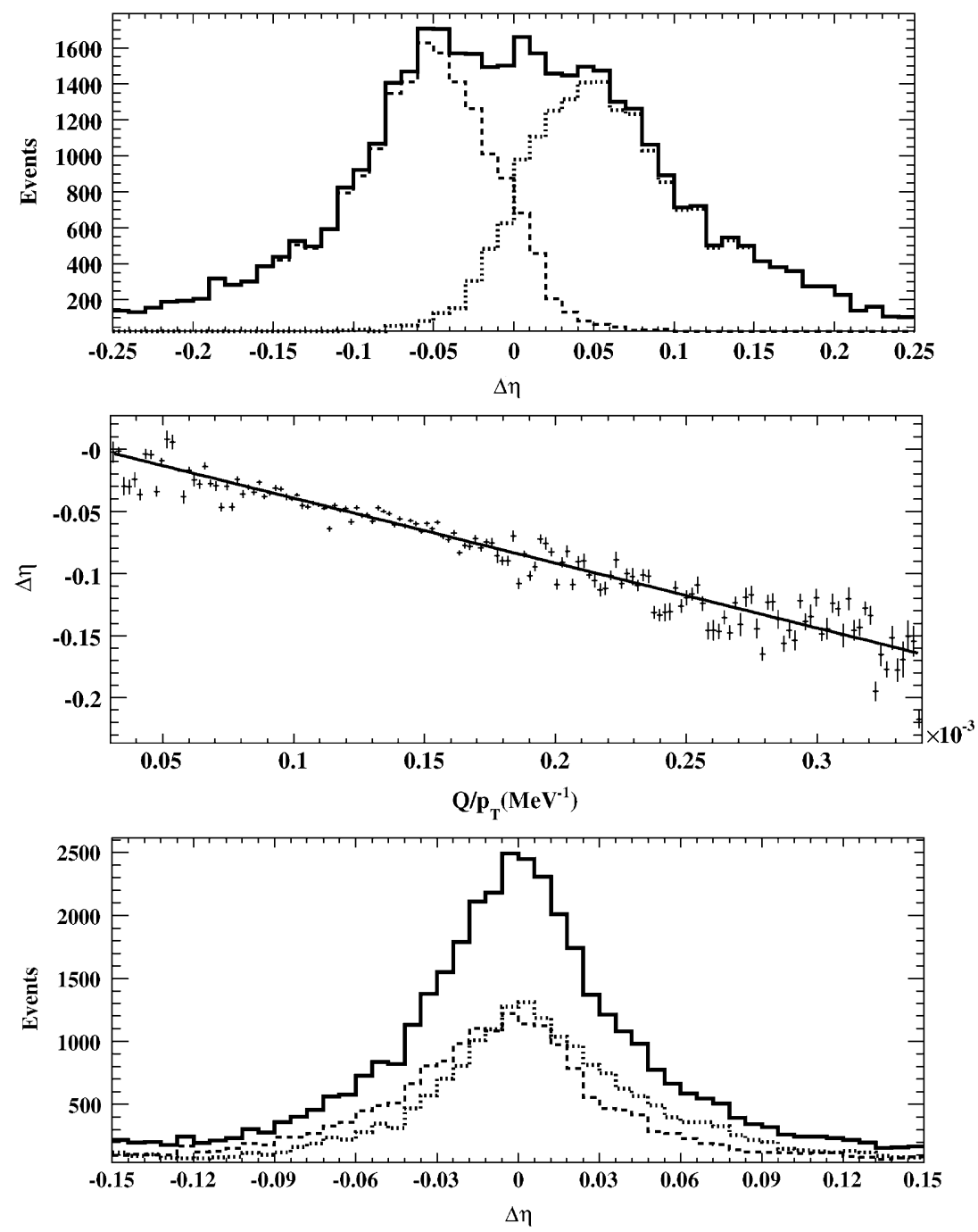

Fig. 2. Extrapolation of ID tracks to the TGC middle station.

TABLE I

AsSOCIATION CUTS ON PROXIMITY OF Digits TO

PARAMETRIZED TRACK TRAGECTORY

\begin{tabular}{||c|c|c||}
\hline \hline MEASUREMENT & $\begin{array}{c}\text { CUT IN } \\
\text { MEASUREMENT } \\
\text { DIRECTION }\end{array}$ & $\begin{array}{c}\text { CUT IN } \\
\text { OTHER } \\
\text { DIRECTION }\end{array}$ \\
\hline MDT & $\Delta \eta<0.06$ & $\Delta \phi<0.5$ \\
\hline CSC $\eta$ & $\Delta \eta<0.06$ & $\Delta \phi<0.5$ \\
\hline TGC wire & $\begin{array}{c}\Delta \eta<0.1 \text { inner station } \\
\text { T }<0.15 \text { middle station }\end{array}$ & $\Delta \phi<0.3$ \\
\hline TGC strip & $\Delta \phi<0.2$ & $\Delta \eta<0.5$ \\
\hline RPC $\eta$ & $\Delta \eta<0.1$ & $\Delta \phi<0.2$ \\
\hline RPC $\phi$ & $\Delta \phi<0.1$ & $\Delta \eta<0.4$ \\
\hline
\end{tabular}

But the level-1 trigger is not sharp and the fraction of muons with $\mathrm{p}_{\mathrm{T}}$ lower than $6 \mathrm{GeV}$ will be collected. Fig. 3 shows the efficiency of the level-1 simulation with $\mathrm{p}_{\mathrm{T}}$ threshold of about $6 \mathrm{GeV}$ as function of $\mathrm{p}_{\mathrm{T}}$. Around $69 \%$ of the events from the datasets mentioned above passed the level-1 trigger simulation. Therefore a signal dataset with $\mathrm{p}_{\mathrm{T}}$ less than $6 \mathrm{GeV}$ has been chosen.

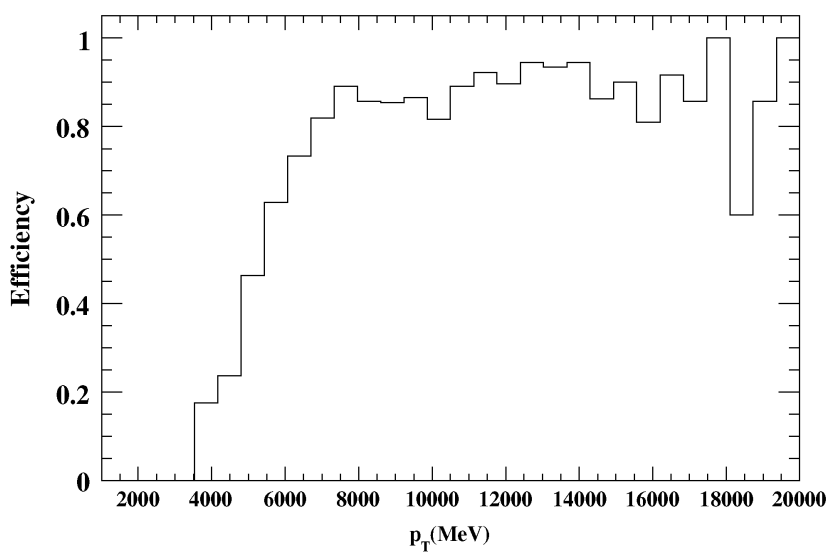

Fig. 3. Level-1 simulation efficiency as function of $\mathrm{p}_{\mathrm{T}}$.

A sample of $\mathrm{bb} \rightarrow \mu \mathrm{X}$, i.e., $\mathrm{b}-\mathrm{bar} \mathrm{b}$ events in which there is a muon with $\mathrm{p}_{\mathrm{T}}>6 \mathrm{GeV}$, was used to represent the background since the dataset in which there is a muon with $\mathrm{p}_{\mathrm{T}}>4 \mathrm{GeV}$ was not simulated yet. The cross section was normalized to represent the background $\mathrm{bb} \rightarrow \mu \mathrm{X}$, where a muon has $\mathrm{p}_{\mathrm{T}}>4 \mathrm{GeV}$. 

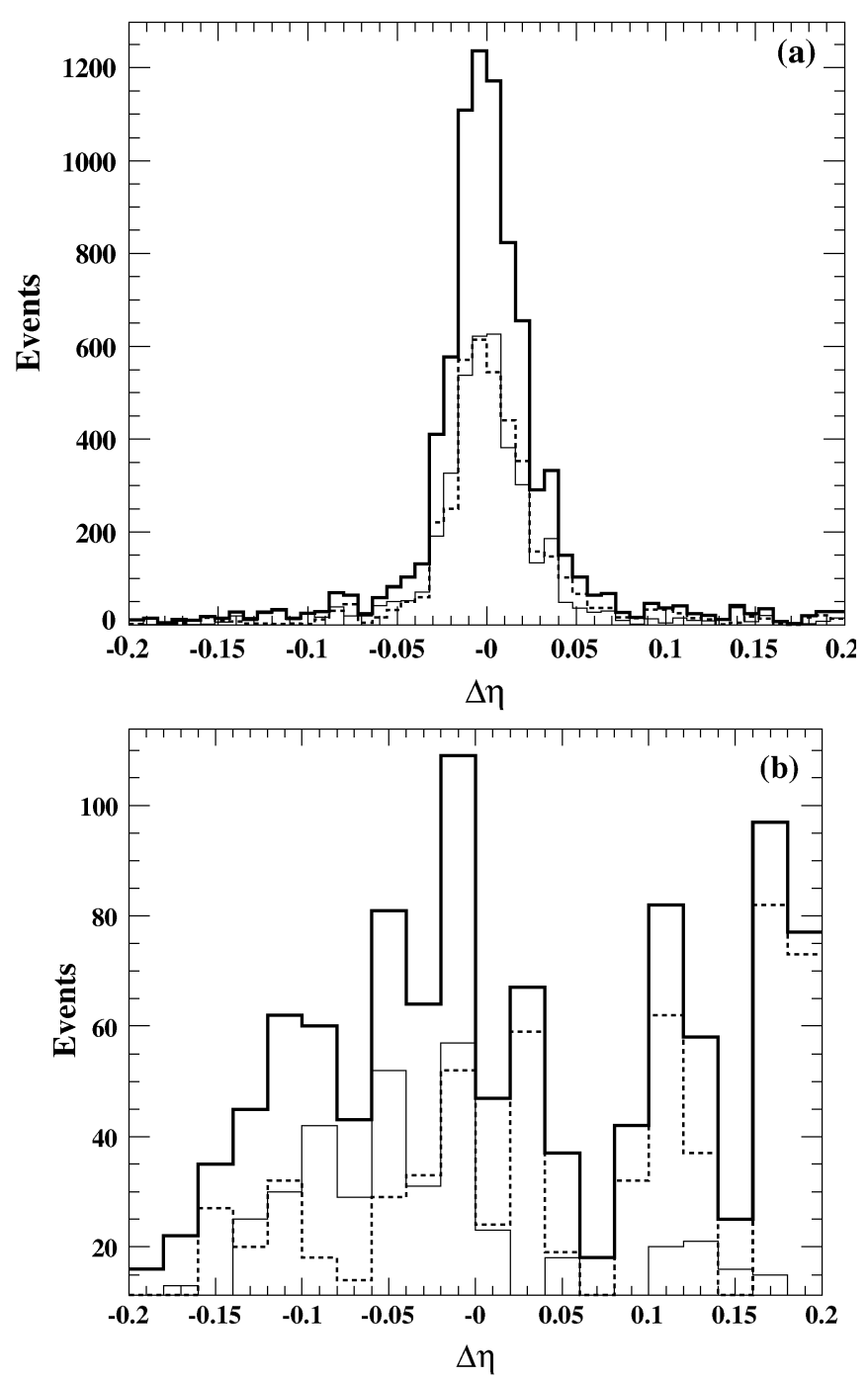

Fig. 4. Distribution of distance in $\eta$ between the ID tracks, extrapolated to MS entrance, and MDT digits for signal (a) and background (b) datasets. The dotted/dashed histogram presents the distributions for muons/anti muons separately, and the black fat histogram is their sum.

In the calculation of purity we used the following cross sections: $\sigma\left(\mathrm{J} / \psi \rightarrow \mu^{+} \mu^{-}\right)=14 \mathrm{nb}$ and $\sigma(\mathrm{bb} \rightarrow \mu \mathrm{X})=5400 \mathrm{nb}$ with the muon $\mathrm{p}_{\mathrm{T}}$ cuts described above.

The samples were produced using the Pythia [9] event generation program. The $\Lambda_{b}$ decay for the signal was generated using the EvtGen [10] program. The interaction of produced particles with the ATLAS detector was simulated using the GEANT4 package [11]. The resulting events were reconstructed with ATLAS reconstruction software release 10.5.0. The level-1 trigger simulation was applied to the samples.

\section{Association Cuts}

Fig. 4 shows the distribution of the distance in $\eta$ between the extrapolated ID track and MS sub detector digits for $\mu^{-}$(black thin line) and $\mu^{+}$(dashed line) for signal (a) and background (b) samples. Using such distributions to guide us, we decided on the association cuts shown in Table I.

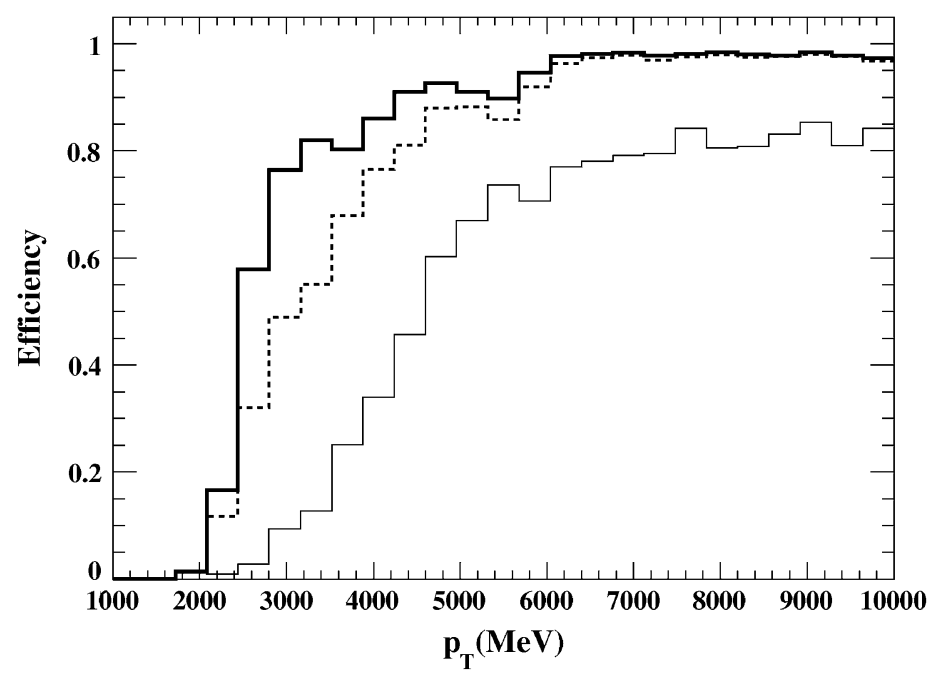

Fig. 5. Muon identification efficiency of the MuId algorithm (thin line), using ID track association to precision chambers digits (bold line) and association to only trigger chambers digits (dashed line) as a function of muon $\mathrm{p}_{\mathrm{T}}$.

\section{Algorithm PerformanCE}

\section{A. Performance for $\mathrm{J} / \psi$ Decays}

Fig. 5 shows the muon identification efficiency of the MuId algorithm and the algorithm described here, when the decision is based on association with precision chambers digits (MDT or CSC) (bold line), or when the decision is based on association with trigger chambers digits (RPC or TGC) (dashed line) as a function of muon $\mathrm{p}_{\mathrm{T}}$. The MuId algorithm reaches its maximal efficiency of about $80 \%$ for a $\mathrm{p}_{\mathrm{T}}$ of about $6 \mathrm{GeV}$. When trying to reconstruct $\mathrm{J} / \psi \rightarrow \mu^{+} \mu^{-}$in the signal sample, this algorithm identifies $30 \%$ of $\mathrm{J} / \psi$ with purity $56 \%$.

The low $\mathrm{p}_{\mathrm{T}}$ algorithm based on association with precision digits reaches the efficiency of about $80 \%$ down to momentum value of about $3 \mathrm{GeV}$. When trying to reconstruct $\mathrm{J} / \psi \rightarrow \mu^{+} \mu^{-}$ using the precision chambers digits, $86 \%$ of $\mathrm{J} / \psi$ are identified with purity about $17 \%$. When the low $\mathrm{p}_{\mathrm{T}}$ decision is based on association with trigger chambers digits (RPC or TGC), as a function of muon $\mathrm{p}_{\mathrm{T}}$, each track is required to have $\eta$ and $\phi$ digits. The trigger chambers are slightly less efficient at $\mathrm{p}_{\mathrm{T}}<4$ $\mathrm{GeV}$, because there are no RPC in the inner station of the barrel, and some muons never reach the middle station. A selection based on trigger chamber information identifies $72 \%$ of $\mathrm{J} / \psi$ with purity $52 \%$.

If we make an inclusive selection, for maximum efficiency, accepting as a muon each candidate that either has a precision chamber digits or has $\eta$ and $\phi$ trigger chambers digits, the resulting efficiency is $88 \%$ but the purity is only $17 \%$. Fig. 6(a) shows the invariant mass distribution of signal and background for this selection. The white histogram shows pairs of real muons from $\mathrm{J} / \psi$, the grey histogram shows fakes and the black are real muons not from $\mathrm{J} / \psi$ decays.

Finally, if we make a tight selection, requiring each candidate to have both precision chamber digits and trigger chamber digits, $66 \%$ of $\mathrm{J} / \psi$ particles are identified with purity $67 \%$. Fig. 6(b) shows the invariant mass distribution of signal and background for this selection. 

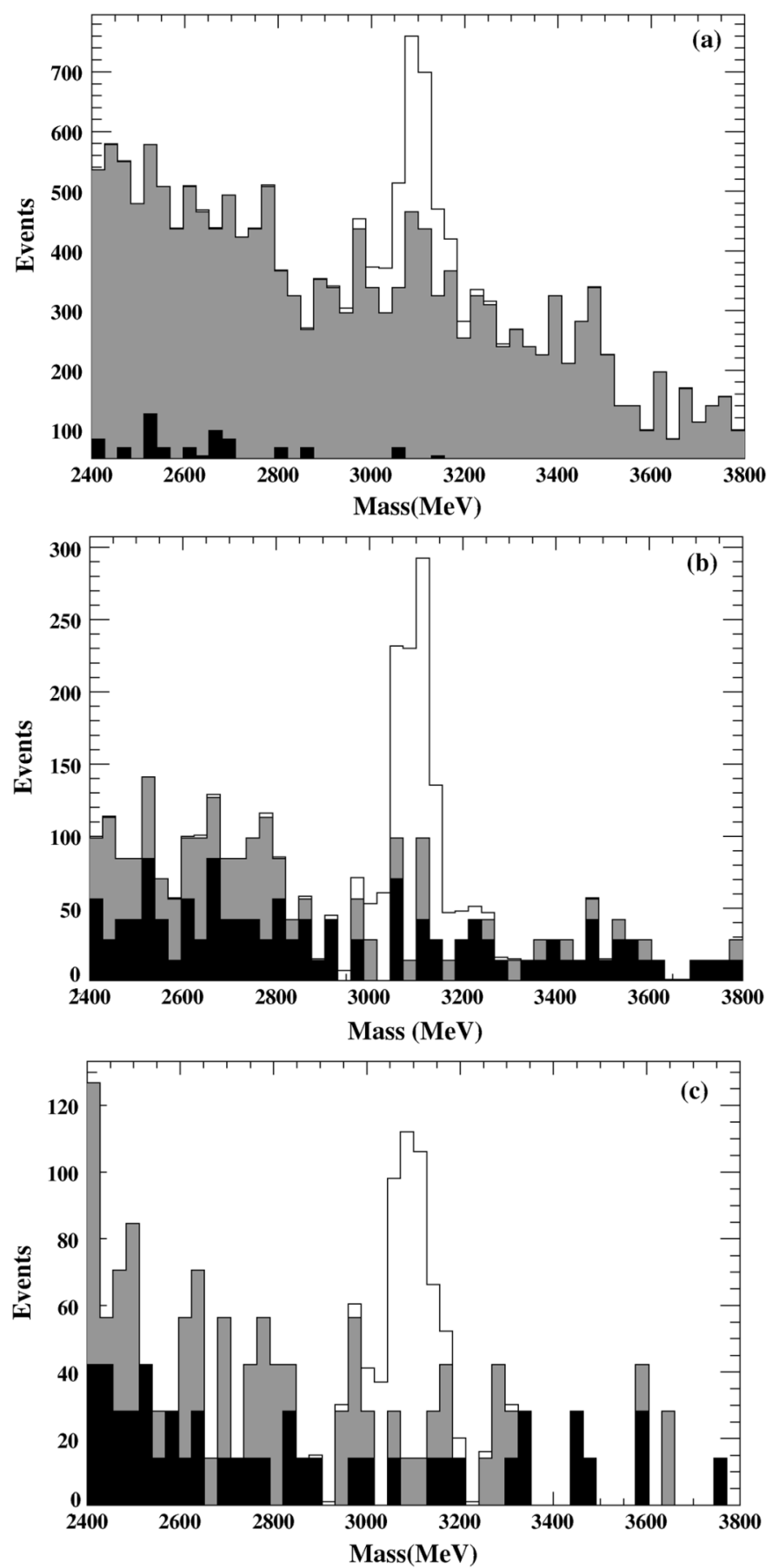

Fig. 6. Invariant mass distribution calculated from two tracks identified as muons by (a) an inclusive selection, (b) a tight selection, and (c) the MuId algorithm. The white histogram shows pairs of real muons from $\mathrm{J} / \psi$, the grey histogram is fakes and the black is real muons but not from $\mathrm{J} / \psi$ decays.

Comparing the invariant mass distributions presented in Fig. 6(a) and (b), we can see that using both precision and trigger digits reduces the number of misidentified tracks (grey), and thus improves significantly the purity of the method. Fig. 6(c) shows the distribution of signal and background using the MuId algorithm.

\section{B. Performance for Single Low $\mathrm{p}_{\mathrm{T}}$ Muons}

Table II presents a comparison between the efficiency of the algorithm described in this paper and the MuId algorithm for
TABLE II

EFFICIENCY FOR INCLUSIVE MUONS IN DIFFERENT SELECTIONS

\begin{tabular}{|c|c|c|c|}
\hline \multirow{2}{*}{ ALGORITHM } & \multicolumn{2}{|c|}{$\mu$ EFFICIENCY } & FAKE \\
\cline { 2 - 3 } & $\mu(2.5) \mu(4)$ & $\mu(6)$ & EFFICIENCY \\
\hline MuId & $58 \%$ & $80 \%$ & $4 \%$ \\
\hline inclusive selection & $94 \%$ & $95 \%$ & $13 \%$ \\
\hline tight selection $^{2}$ & $81 \%$ & $88 \%$ & $4 \%$ \\
\hline trigger selection $^{2}$ & $85 \%$ & $91 \%$ & $7 \%$ \\
\hline
\end{tabular}

I The muon efficiency was calculated using two data samples, the first where one muon has $\mathrm{p}_{\mathrm{T}}>4 \mathrm{GeV}$ and the second muon has $\mathrm{p}_{\mathrm{T}}>2.5 \mathrm{GeV}$ and the second sample where at least one muon has $\mathrm{p}_{\mathrm{T}}>6 \mathrm{GeV}$.

2 The trigger selection is based on association with trigger chambers digits (RPC or TGC)

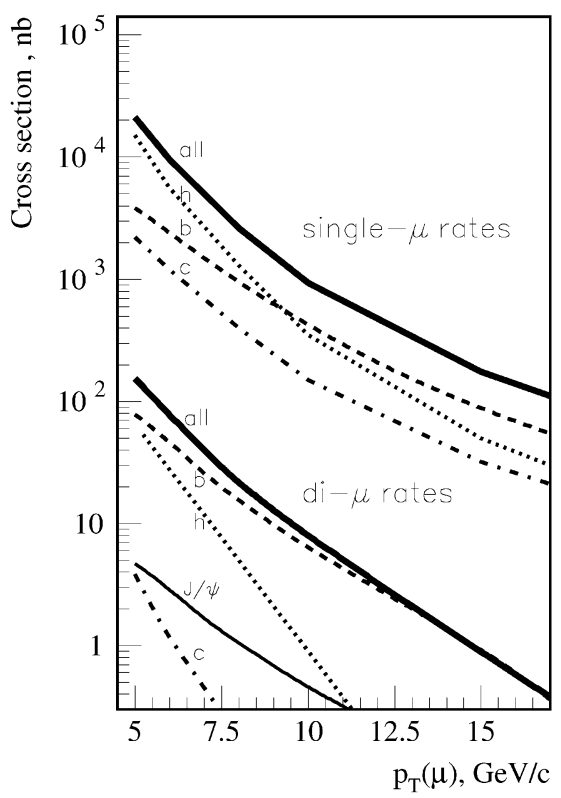

Fig. 7. Invariant mass distribution calculated from two tracks identified as muons by (a) an inclusive selection, (b) a tight selection and (c) the MuId algorithm. The white histogram shows pairs of real muons from $\mathrm{J} / \psi$, the grey histogram is fakes and the black is real muons but not from $\mathrm{J} / \psi$ decays.

identifying any muon. The fake rate is calculated from all reconstructed tracks with $\mathrm{p}_{\mathrm{T}}$ above $2.3 \mathrm{GeV}$. The efficiency for low $\mathrm{p}_{\mathrm{T}}$ muons can be improved by $40 \%$ with respect to MuId, when using a tight selection with the same fake rate. Using an inclusive selection the efficiency is very high, but the fake rate is also higher than with MuId. For muons with $\mathrm{p}_{\mathrm{T}}>6 \mathrm{GeV}$ the differences in efficiency are less significant.

\section{AdAPTATION FOR THE SECOND LeVEl TRIGGer}

Thanks to its simplicity this algorithm can be used in the level-2, in addition to the usage in the offline reconstruction.

The next goal of this work will be the development of a level-2 trigger algorithm that increases the number of events that are interesting for B physics via identification of relatively low $\mathrm{p}_{\mathrm{T}}$ muons from $\mathrm{J} / \psi$ decays. Our algorithm is suitable for the trigger because there is no complicated pattern recognition and fitting procedure involved.

The selection of the level-1 trigger is expected to retain $23 \mathrm{kHz}$ events at a luminosity of $2 \times 10^{33} \mathrm{~cm}^{-2} \mathrm{~s}^{-1}$ where one muon with $\mathrm{p}_{\mathrm{T}}$ threshold of about $6 \mathrm{GeV}$. Only $4 \mathrm{KHz}$ of these 

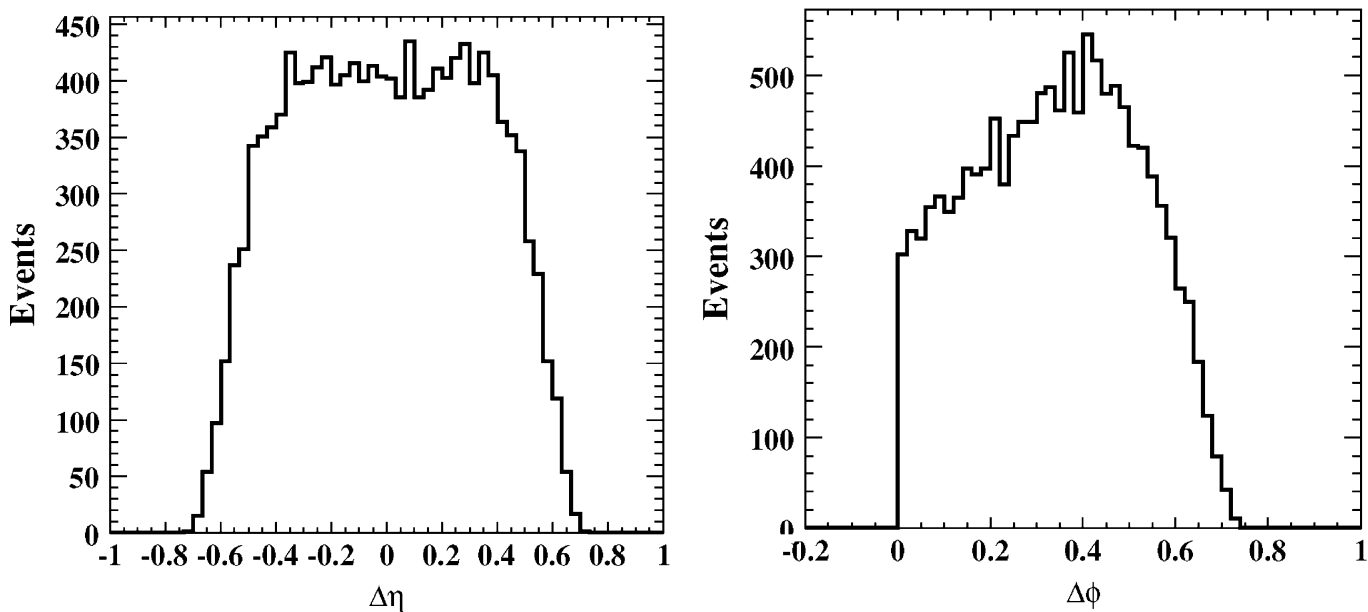

Fig. 8. Angular distance in $\eta$ (left) and $\phi$ (right) between two muons from $\mathrm{J} / \psi$ decay.

are $\mathrm{b}$ events, with most others either muons from $K / \pi$ decays or cavern background.

At the level-2 trigger the rate must be reduced by a factor 100 , so could lose many more b events. Rather than be left with an unbiased fraction of muons with $\mathrm{p}_{\mathrm{T}}>6 \mathrm{GeV}$, we would like to be efficient for "gold" channels $(\mathrm{J} / \psi)$ at level-2.

Fig. 7 compares the expected single muon rates to di-muon and $\mathrm{J} / \psi$ rates at a luminosity of $2 \times 10^{33} \mathrm{~cm}^{-2} \mathrm{~s}^{-1}$. For di-muons the horizontal axis shows the $\mathrm{p}_{\mathrm{T}}$ of the lower $\mathrm{p}_{\mathrm{T}}$ muon. A di-muon trigger will allow for an effective selection of channels with $\mathrm{J} / \psi \rightarrow \mu^{+} \mu^{-}$.

Level-2 trigger algorithms start from Regions of Interest (ROI) found by the level-1 trigger. The description of the RoI mechanism can be found in [7]. In order to find $\mathrm{J} / \psi$ decays we open a search window around the direction of the muon ROI. Fig. 8 shows the distribution of angular distance in $\eta$ and $\phi$ between two muons from $\mathrm{J} / \psi$ decays. We find ID tracks in the search region using the trigger-tracking program IdScan. The description of IdScan algorithm can be found in [12]. We read out all MS sub detectors in the region around each selected track and associate MS digits with the ID track.

This part of the development of the algorithm is at an early stage, but its potential for improving the ATLAS trigger for $b$ physics is very significant.

\section{CONCLUSION}

We have developed an algorithm for identifying low $\mathrm{p}_{\mathrm{T}}$ muons with the ATLAS detector. The user may select the purity and efficiency of her/his sample by simple cuts on the algorithm output. This algorithm provides significantly better efficiency for identifying muons with $\mathrm{p}_{\mathrm{T}}$ below $5 \mathrm{GeV}$ than the already existing algorithm, without compromising the purity of the resulting muon sample.

\section{REFERENCES}

[1] ATLAS Inner Detector Tech. Design Rep. ATLAS Collaboration, Apr. 1997, CERN/LHCC 97-16, CERN/LHC 97-17.

[2] ATLAS Muon Spectrometer Tech. Design Rep. ATLAS Muon Collaboration, Jun. 1997, CERN/LHCC 97-22.

[3] ATLAS Computing Tech. Design Rep. ATLAS Computing Group, CERN-LHCC-2005-022.

[4] D. Adams, "Track reconstruction in the ATLAS muon spectrometer with MOORE," ATLAS Note, ATL-SOFT-2003-007, 2003.

[5] Th. Laugouri, A Muon Identification and Combined Reconstruction Procedure for the ATLAS Detector at the LHC at CERN Dec. 2004, vol. 51, no. 6, pt. 1, pp. 3030-3033.

[6] R. Clifft and A. Poppleton, "IPATREC: Inner detector pattern-recognition and track fitting," ATLAS Internal Note, ATLAS-SOFT-94-009, 1994.

[7] ATLAS High-Level Trigger Data Acquisition and Controls June 2003, vol. 30, CERN/LHCC/2003-022.

[8] G. Usai, Feasibility Study of a LVL-2 Low Di-Muon Trigger Based on Tile Calorimeter. Geneva, Switzerland, Jan. 13, 2003, ATL-DAQ2003-017, p. 43, CERN

[9] T. Sjostrand, P. Eden, C. Friberg, L. Lonnblad, G. Miu, S. Mrenna, and E. Norrbin, Computer Phys. Commun., vol. 135, p. 238, 2001.

[10] A. Ryd, EvtGen A Monte Carlo Generator for B-Physics vol. 462, pp. 152-155, 2001, NIM A

[11] S. Agostinelli, Geant4-A Simulation Toolkit vol. 506, pp. 250-303, 2003, NIM A.

[12] H. Drevermann and N. Konstantinidis, "Algorithm to select space points of tracks from single primary interactions in ATLAS," ATLAS Internal Note, ATL-COM-DAQ-2003-040, 2003. 